\title{
High-precision measurement of isotopic fission yields of ${ }^{236} \mathrm{U}^{*}$
}

\author{
L. Audouin ${ }^{1, \star}$, J. Taieb², A. Chatillon ${ }^{2}$, L. Grente ${ }^{2}$, G. Boutoux ${ }^{2}$, T. Gorbinet ${ }^{1}$, J.-F. Martin ${ }^{2}$, \\ H. Alvarez-Pol ${ }^{6}$, Y. Ayyad ${ }^{6}$, G. Bélier ${ }^{2}$, J. Benlliure ${ }^{6}$, M. Caamaño ${ }^{6}$, E. Casarejos ${ }^{5}$, D. Cortina- \\ Gil $^{6}$, F. Farget ${ }^{8}$, B. Fernández-Domínguez ${ }^{6}$, A. Heinz ${ }^{7}$, H. Johansson ${ }^{7}$, B. Jurado ${ }^{3}$, A. Kelić- \\ $\mathrm{Heil}^{4}$, N. Kurz ${ }^{4}$, B. Laurent ${ }^{2}$, S. Lindberg ${ }^{7}$, B. Löher ${ }^{4}$, C. Nociforo ${ }^{4}$, C. Paradela ${ }^{6}$, S. Pietri ${ }^{4}$, \\ D. Ramos ${ }^{6}$, J.-L. Rodriguez-Sanchez ${ }^{6}$, C. Rodrìguez-Tajes ${ }^{8}$, D. Rossi ${ }^{4}$, K.-H. Schmidt ${ }^{4}$, \\ H. Simon ${ }^{4}$, L. Tassan-Got ${ }^{1}$, H. Törnqvist ${ }^{4}$, B. Voss ${ }^{4}$, H. Weick ${ }^{4}$, and Y. Yan ${ }^{1}$ \\ ${ }^{1}$ Institut de Physique Nucléaire d'Orsay, Université Paris-Sud, CNRS, IN2P3, 91406 Orsay, France \\ ${ }^{2}$ CEA, DAM, DIF, 91297 Arpajon, France \\ ${ }^{3}$ CENBG, Université de Bordeaux, CNRS, IN2P3, 33175 Gradignan, France \\ ${ }^{4} \mathrm{GSI}$, Planckstr. 1, 64291 Darmstadt, Germany \\ ${ }^{5}$ University of Vigo, 36310 Vigo, Spain \\ ${ }^{6}$ Dpt. of Particle Physics, University Santiago de Compostela, 15782 Santiago de Compostela, Spain \\ ${ }^{7}$ Chalmers University of Technology, 41296 Gothenburg, Sweden \\ ${ }^{8}$ CNRS, GANIL, Bd. H. Becquerel, 14076 Caen, France
}

\begin{abstract}
We report on the second SOFIA experiment, dedicated to the fission yields of ${ }^{236} \mathrm{U}^{*}$, analog to ${ }^{235} \mathrm{U}(\mathrm{n}, \mathrm{f})$. The measurement is based on the inverse kinematics method, using a relativistic, secondary beam of ${ }^{236} \mathrm{U}$. Both fission fragments are identified in mass and charge in the SOFIA recoil spectrometer. The obtained isotopic yields are compared with existing spectroscopy measurements and the elemental yields are used to discuss the treatment of the even-odd effect with energy in nuclear data libraries.
\end{abstract}

\section{Introduction}

The study of fission fragment yields impacts is of interest for both basic and applied science. In the nuclear energy field, fission yields directly impact the reactor neutronics: they are the source of the delayed neutrons and some of them act as neutron poisons. In the case of a criticality excursion, the quick, local accumulation of the fragments may change drastically the local parameters. Furthermore, fission fragments are the main source of the residual power and radioactivity. Therefore, a precise knowledge of their production is of primary importance for the development of predictive models to simulate the decay heat after a core shutdown or the neutron flux. Additionally, their amount in the fuel is one of the main dimensioning parameters for the shielding in the handling and reprocessing of the used fuel, for the safety analysis in a loss of coolant situation, and for the early storage cooling.

Fission fragments are also a remarkable probe for nuclear structure. Many questions are still opened, like the exact role of the proton and neutron shells on the partition, the influence of shells at large deformation, the influence of the pairing (which is responsible for the even-odd staggering

\footnotetext{
^e-mail: audouin@ipno.in2p3.fr
} 
in the yields), or the distribution of the fission energy among the fragments and their deformation at scission.

Despite several decades of study, experimental knowledge of the fission yields remains largely incomplete. The difficulties, and more often than not the impossibility, to make targets of mid- or short-lived nuclides is the main reason for this. But even for easily-available isotopes, the experimental information is only partial. Over the last decades, the mass yields have been measured with a high precision, the Lohengrin spectrometer [1] being the prime tool for such experiments. Still, isotopic information is scarce. Even with state-of-the-art techniques, the very low kinetic energy of the fragment only allows for an estimation of the charge of the light fragment. Gamma spectroscopy can be used as an alternative or complementary technique, but it provides results on a limited number of fragments with well-known decay chains, and the normalization of the results can be challenging.

The inverse kinematics has opened new experimental perspectives. A secondary beam of radioactive nuclei can be formed by a first reaction, allowing for the study of a large range of fissioning systems; and the kinematic boost provides the fragments with a large kinetic energy, thus making the measurement of the nuclear charge possible with a good (even excellent) resolution. After the pioneering experiment of K.-H. Schmidt [2] at GSI, in which all fission fragments were identified in charge (but not in mass) for the first time, this method has been successfully applied at GSI by the SOFIA collaboration [3] at relativistic energies, and at GANIL [5] at Coulomb-barrier energy, this time allowing the simultaneous measurement of mass and charge of all fission fragments.

In this paper, we report on the second SOFIA experiment, dedicated to the fission yields of ${ }^{236} \mathrm{U}^{*}$. These data are of primary relevance for the nuclear fuel cycle, since the fission of ${ }^{236} \mathrm{U}^{*}$ is the analog of the ${ }^{235} \mathrm{U}(\mathrm{n}, \mathrm{f})$, an essential reaction for most current reactors.

\section{The SOFIA setup}

The SOFIA setup has been designed to identify in charge and mass, with a very high resolution, both fission fragments simultaneously.

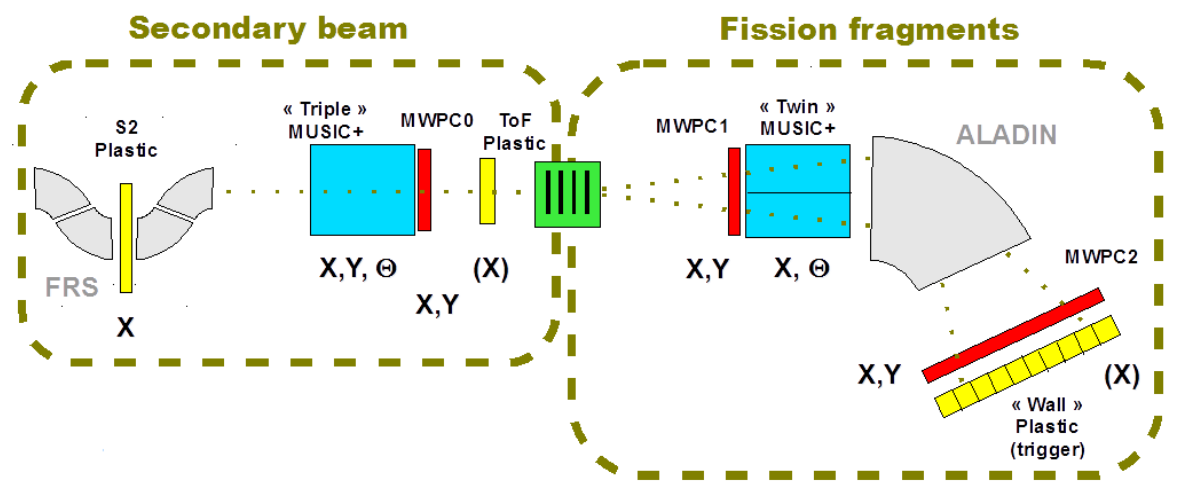

Figure 1. Scheme of the SOFIA setup. The left part is dedicated to the identification of the secondary beam. The active target is the fission location. The right part is dedicated to the fission fragments.

The nuclide of interest is formed by fragmentation of a $1 \mathrm{~A} \mathrm{GeV}{ }^{238} \mathrm{U}$ beam. It is selected in the FRS spectrometer [6], which is also used for the identification in mass and charge by the $B \rho-T o F-\Delta E$ method (see the left part of figure 1). The presence of charge states makes this identification very 
challenging and motivated the design of a specific ionization chamber, the "Triple-MUSIC", which basically consists of 3 independent, successive ionization chambers separated by stripping foils, thus increasing dramatically the probability of detecting nuclei without any electron attached.

The fission is triggered by electromagnetic interaction in an "active target" which consists of 2 uranium and 1 lead targets, supplemented by an aluminum target that is used to suppress the contribution of the nuclear collisions to the measured yields (see [3] for a detailed explanation of this correction). The choice of the Coulomb mechanism as the fission trigger is motivated by the large cross section of this interaction (several barns), and the moderate excitation energy deposited in the system (14.5 MeV on average, which is equivalent to neutrons of $8.2 \mathrm{MeV}$ interacting with a ${ }^{235} \mathrm{U}$ nuclei). The downside is that there is no way to determine the excitation energy event-by-event.

The part of the setup dedicated to the fission fragments (right part of figure 1) can be summed-up as a large-acceptance recoil spectrometer, built around the ALADIN magnet. The identification of the fragments is realized using the $B \rho-T o F-\Delta E$ method. The nuclear charge is deduced from the energy loss in a segmented ionization chamber ("Twin-MUSIC"), which also measures the horizontal position and angle. The tracking is completed by Multi-Wires Proportional Counters. The time of flight (ToF) is measured by plastic scintillators, with a flight path of about $8 \mathrm{~m}$.

\section{Identification of the secondary beam and of fission fragments}

As already mentioned, the identification of the secondary beam is complicated by the chances of ions to keep one or even two electrons in the FRS and the detectors. To overcome this issue, the ionization chamber is divided into 3 sections separated by stripping foils, leading to 3 independent measurements and bringing the chance of correct identification close to $100 \%$. This is illustrated on the left picture in figure 2 . The misidentified ions can be clearly pointed out since the A/q separation is very good for fragments close to the projectile: they are located between the 2 protactinium isotopes and amount to less than a percent of the ${ }^{236} \mathrm{U}$ (more details on the analysis technique are presented in [4]).

The measurement of the nuclear charge of the fission fragments brings unprecedented results, with a resolution of 0.35 charge FWHM. As it can be seen on figure 2, the charge separation is practically complete. The mass resolution is also very good, varying between 0.55 and 0.7 mass FWHM (corresponding to light and heavy fragments, respectively).
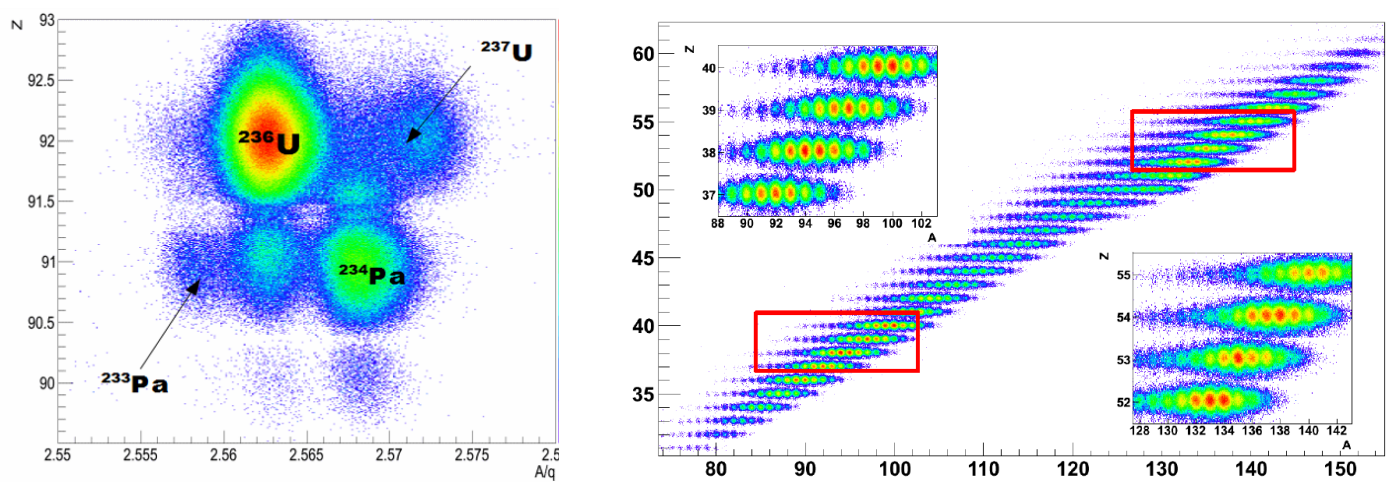

Figure 2. Identification matrix (A,Z) of the ${ }^{236} \mathrm{U}$ secondary beam (left) and of fission fragments of ${ }^{236} \mathrm{U}^{*}$ (right). Only a very limited quantity of other nuclides are transmitted along ${ }^{236} \mathrm{U}$. 
Despite a very short measurement (20h of data taking), more than 2 million of fissions of ${ }^{236} \mathrm{U}^{*}$ were measured, so that the statistical uncertainty on the elemental yields is below $0.5 \%$ for the mostproduced isotopes, and below $1.5 \%$ for the less abundant fragments.

\section{Comparison of yields with existing data and libraries}
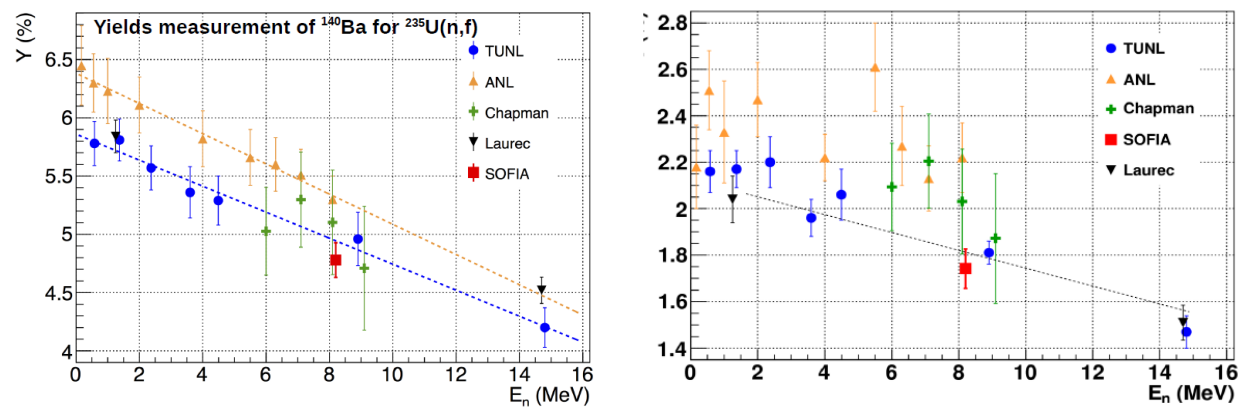

Figure 3. Comparison between SOFIA results and yields obtained by gamma spectroscopy for ${ }^{140} \mathrm{Ba}$ (left) and ${ }^{147} \mathrm{Nd}$ (right). The distribution of the excitation energy for SOFIA is not included on the plot, since it is rather large and non-gaussian. However the average value is very well controlled thanks to a comparison with the mass distributions of the fragments for neutron-induced reactions at various energies (see [4] for more details).

As already mentioned, spectroscopic measurements can provide data on the yield of some particular isotopes. They are well-suited for mono-energetic measurements, hence can be used for studying the dependence of yields on the energy, but the results depend on the knowledge of the branching ratios and can prove challenging to normalize. Conversely, SOFIA brings completely-independent yields, with a very good confidence on the normalization due to the very high detection efficiency (about 90\%), but for an averaged energy spectrum. On figure 3 we compare the SOFIA results with spectroscopic data from Laurec [7], Chapman [8], TUNL [9] and ANL [10]. For ${ }^{140} \mathrm{Ba}$, our results are very consistent with TUNL and Chapman, and seem to point a normalization issue in the work of ANL (and possibly of Laurec). For ${ }^{147} \mathrm{Nd}$, we have once more a good consistency with TUNL and a clear normalization disagreement with ANL. This time the agreement is satisfactory with Laurec, but not with Chapman.
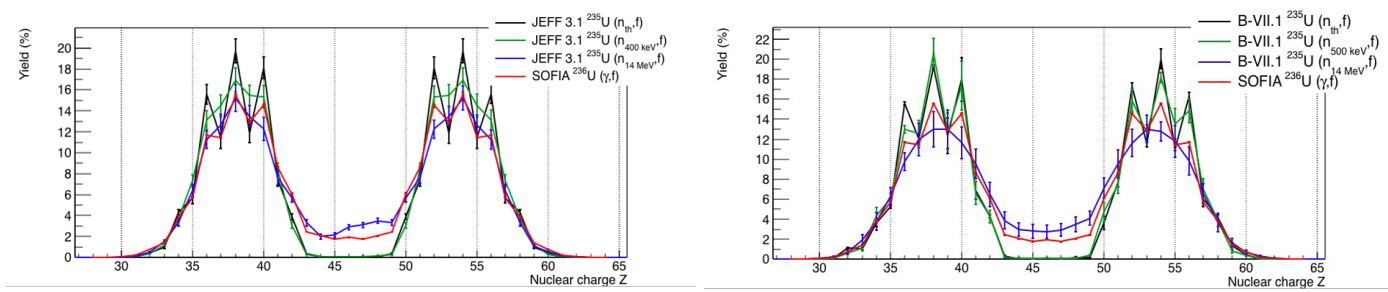

Figure 4. Comparison between experimental elemental yields and the ENDF and JEFF libraries.

The comparison of the elemental yields with libraries such as JEFF-3.1 and ENDF-B-VII.1, presented in figure 4 , reveals a general consistency, but also some significant differences. The libraries 
provide data at thermal energy, 400 and $500 \mathrm{keV}$ respectively, and $14 \mathrm{MeV}$. SOFIA data are measured with an equivalent neutron energy of $8.2 \mathrm{MeV}$, so they are expected to lay in between the last two series. This is indeed the case, but the comparison reveals that the dampening of the even-odd effect is very different from one library to the other. In ENDF, the $500 \mathrm{keV}$ points present a moderate dampening with respect to thermal and the evolution toward the SOFIA energy seems coherent, with the exception of $\mathrm{Z}=55$. However, the complete dampening of the even-odd staggering at $14 \mathrm{MeV}$ is very sudden compared to SOFIA energy, and could be questioned. In the case of JEFF, the dampening appears very inconsistent with our measurement, with odd elements being clearly overestimated at $400 \mathrm{keV}$.

\section{Total kinetic energy and prompt neutron yields}
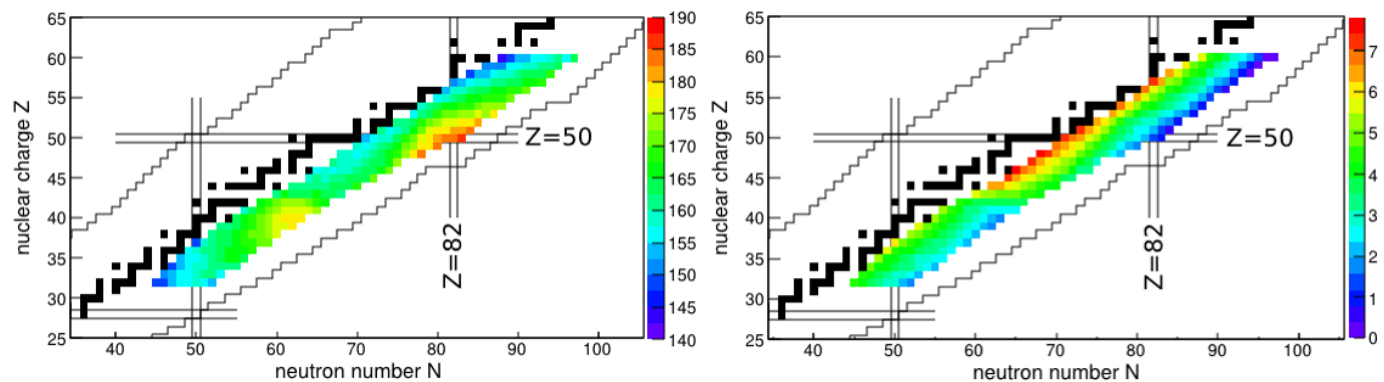

Figure 5. Total Kinetic Energy (left plot, vertical axis in $\mathrm{MeV}$ ) and average prompt neutron multiplicity (right plot, vertical axis in neutron number) as a function of $\mathrm{Z}$ and $\mathrm{N}$ of each fission fragment.

The total kinetic energy (TKE) of each fragment can be calculated based on the precise measurement of the velocity. It is interpreted as an indication of the deformation of the fragments at scission: (quasi-)spherical nuclei will contribute to a higher Coulomb repulsion than deformed ones. This explanation is clearly corroborated by figure 5, in which the TKE is plotted for each isotope (left plot). An island of larger TKE in the immediate vicinity of the doubly-magic ${ }^{132} \mathrm{Sn}$ is clearly visible. The symmetric fission presents a significantly lower TKE, confirming that the fragments are formed in a more elongated configuration. The precise measurement of the TKE isotope per isotope is a sensitive tool to test scission models.

Since both fission fragments and the fissioning system are identified in mass, it is possible to extract the neutron multiplicity on an event-by-event basis. The mean value $(\bar{v})$ is plotted for each isotope on picture 5 (right plot). The overall trend is an increase of $\bar{v}$ with the proximity of the valley of stability, which could seem counter-intuitive since the neutron separation energy increases along this direction. Actually, the driving force of the neutron multiplicity is rather the deformation of the prefragments: the deformation energy will relax into excitation energy, which in turn will lead to neutron emission. This explanation is corroborated by the antisymmetry one can observe between the TKE and the $\bar{v}$ plots.

\section{Summary and oulook}

Fission fragments from ${ }^{236} \mathrm{U}^{*}$ have been simultaneously identified both in mass and charge with an excellent resolution using the SOFIA setup at a mean excitation energy of $14.5 \mathrm{MeV}$. Very accu- 
rate isotopic yields have been obtained, with a resolution varying from 2.5 to $7 \%$ (less than $2 \%$ for elemental yields).

Isotopic yields are found consistent with recent spectroscopic measurements and indicate a normalization problem with a previous set of data. SOFIA data provide a stringent text from nuclear libraries. For example, a significant discrepancy is found in the treatment of the dampening of the even-odd elemental staggering by the JEFF-3.1 library.

A new experiment is being prepared, in which the SOFIA set-up will be coupled with R3B [11] detectors such as the CALIFA calorimeter, the GLAD magnet and the NEULAND neutron detector. This experiment will investigate the fission of very neutron-deficient nuclides in the Hg-Bi region, where asymmetric fission is becomes dominant. We are also working on the development of a primary beam of ${ }^{242} \mathrm{Pu}$, which would make possible the accurate measurement of the fission yields of $\mathrm{Np}, \mathrm{Pu}$ and Am isotopes.

\section{References}

[1] W. Lang et al., Nucl. Phys. A 345, 34 (1980)

[2] K.-H. Schmidt et al., Nucl. Phys. A 665, 221-267 (2000)

[3] E. Pellereau et al., Phys. Rev. C 95, 054603 (2017)

[4] Y. Yan, "Study of fission of exotic actinides by relativistic reactions", $\mathrm{PhD}$ thesis, University Paris-Saclay (2016)

[5] C. Rodríguez-Tajes et al., Phys. Rev. C 89024614 (2014)

[6] H. Geissel et al., Nucl. Instrum. Methods B 70, 286 (1992)

[7] J. Laurec et al., Nuclear Data Sheets 111, 2965 (2010)

[8] T. C. Chapman et al., Phys. Rec. C 17, 1089 (1978)

[9] M. E. Gooden et al., Nuclear Data Sheets 131, 319 (2016)

[10] L.E. Glendenin et al., Phys. Rev. C 24, 2600 (1981)

[11] R3B web site 